\title{
An Efficient Power Constrained Transmission Scheme for Hybrid OW/RF Systems
}

\author{
Nestor D. Chatzidiamantis*, Leonidas Georgiadis*, Harilaos G. Sandalidis ${ }^{\dagger}$ and George K. Karagiannidis* \\ *Department of Electrical and Computer Engineering, Aristotle University of Thessaloniki, Greece, Emails:\{nestoras,leonid,geokarag\}@ auth.gr \\ $\dagger^{\dagger}$ Department of Computer Science and Biomedical Informatics, University of Thessaly, Greece, Email:sandalidis@ucg.gr
}

\begin{abstract}
This paper investigates link layer transmission schemes for hybrid optical wireless (OW)/radio frequency (RF) systems with constraints on both per-link and total power consumption at the transmitter. Assuming timeslot structure and using a queue for storing the random data packet arrivals, we formulate a stochastic optimization problem where intelligent control decisions are taken by the hybrid transmitter in each slot, regarding the number of the packets admitted in its queue and the power levels used in each link. The aim of this formulation is to design a control policy that maximizes the transmitter throughput, while satisfying its power constraints as well. A solution is offered by using the Lyapunov optimization framework. An on-line transmission algorithm is derived, which takes control decisions based only on the status of the transmitter queue and the erasure probabilities of the OW and RF links. The proposed algorithm meets the desired throughput objective by efficiently exploiting both links, while provides explicit power consumption guarantees.
\end{abstract}

\section{INTRODUCTION}

Operating at unlicensed optical frequencies, optical wireless (OW) systems offer the potential of broadband capacity [1]. However, OW systems are susceptible to atmospheric effects, which limit their availability. A prominent way that has been suggested for combating these effects is the use of a licencefree radio frequency $(\mathrm{RF})$ channel in conjunction with a $\mathrm{OW}$ channel. The incentive for implementing such hybrid OW/RF systems is that both channels are not affected in the same way by atmospheric effects [2].

The complementary behavior of RF and OW channels has led to the development of multiple transmission techniques suitable for hybrid OW/RF systems (see [3] and the references therein). However, only few of these techniques dealt with link layer issues, i.e., how to efficiently transmit streams of data which are divided into packets and protected through a cyclic redundancy check (CRC) code, through the hybrid OW/RF channel. In [2], a commercially available transmission technique was presented where the same packets are transmitted through both links but only the packets that are deemed more reliable are kept at the receiver. Despite its minimal feedback requirements (when the packets are corrupted from both links), this scheme does not fully exploit the hybrid OW/RF channel, since the maximum throughput that can be achieved is limited to the maximum throughput between the two links. In order to address this issue, an efficient packet coding scheme based on Raptor codes was implemented in [4]. By continuously sending encoded packets through both OW and RF links, this coding scheme was shown to increase the achievable throughput near the capacity limits of the hybrid OW/RF channel, requiring limited feedback. However, any restrictions that may exist in the transmitter total power consumption are not considered in its design.

The assumption of infinite total power resources at the transmitter is not always valid. For example, power restriction issues arise when hybrid $\mathrm{OW} / \mathrm{RF}$ links are used in wireless sensor networks [5], [6] or for the communication between moving entities (such as unmanned aerial vehicles or satellites) and fixed-point stations. In this case, not only the restrictions on the transmitted power of each link, imposed from hardware constraints and safety regulations, but also the limitations on the total power resources of the transmitter, must be taken into account.

In this paper, we investigate transmission schemes that can be applied in hybrid OW/RF transmitters with limited total power resources. Assuming a timeslot structure and using a queue for storing the random data packet arrivals, we formulate a stochastic optimization problem where intelligent per-slot control decisions are taken at the transmitter, regarding the number of the packets admitted in the queue and the power levels used in each link. The objective is to maximize the achievable throughput, while satisfying both the per-link transmission and total power constraints imposed to the hybrid transmitter. A solution to this optimization problem is offered using the framework of Lyapunov Optimization techniques [7]. An on-line control algorithm is developed that dynamically adapts the control decisions based only on the status of the queue and the erasure probabilities of the OW and RF links. The performance of the proposed algorithm is investigated in terms of throughput and average queue backlog, and analytical bounds are derived which illustrate the optimality of the proposed transmission policy.

\section{SySTEM MODEL}

\section{A. Transceiver Design}

The system model under consideration is depicted in Fig. 1. In particular, we consider a hybrid OW/RF transmitter with bidirectional links, which is composed of an admission control mechanism that determines which packets will be transmitted or discarded, a queue which stores the packets to be transmitted, a serial-to-parallel converter that divides the incoming sequence of packets into two subsequences according to the resource allocation control decisions of the transmitter, and the OW and RF transmission subsystems that 


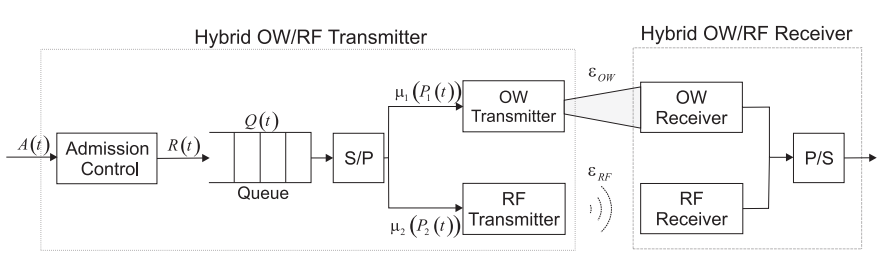

Fig. 1. The system model under consideration.

are used for the packet transmission. At the receiver's end, the packets that are received correctly by each subsystem are inserted into a parallel-to-serial converter which composes the original packet sequence. Since correctly received packets are determined through the CRC code that is applied to each packet, both OW and RF links can be modeled as erasure channels with erasure probabilities $\epsilon_{O W}$ and $\epsilon_{R F}$ respectively [4]. Next, we assume that these probabilities are known at the transmitter.

\section{B. Timeslot Structure}

We assume that the transmitter operates over a slot based structure. Namely, the timeline is divided into successive nonoverlapping slots, where the slot is denoted as $t=0,1,2, \ldots$ and corresponds to the time interval $[t, t+1) ; t$ and $t+1$ are called the "beginning" and the "end" of the slot, respectively. Moreover, we assume that the channel conditions that affect the successful packet transmission in both links remain constant within a transmission slot, while they change in an independently and identically distributed (i.i.d.) fashion in the next slot. In each slot, new packets arrive at the transmitter according to an i.i.d. arrival process $A(t)$ with mean rate $\lambda$ packets per frame and $A(t) \leq A_{\max }$, where $A_{\max }$ is a finite constant.

\section{Power Constraints}

Let $P_{1}(t)$ and $P_{2}(t)$ denote the power levels used for transmission in the OW and RF subsystems in slot $t$. These power allocations are chosen from the sets $\mathcal{P}_{1}$ and $\mathcal{P}_{2}$, satisfying maximum instantaneous power constraints imposed from hardware limitations. Hence,

$$
P_{1}(t) \leq P_{1, \max } \text { and } P_{2}(t) \leq P_{2, \max },
$$

where $P_{1, \max }$ and $P_{2, \max }$ represent the maximum power levels used for transmission in OW and RF subsystems respectively.

Furthermore, we seek transmission policies that select power allocations that are subject to long-term average constraints, imposed from safety regulations and the limitation on the total power consumption of the hybrid transmitter. Hence, the following constraints also need to be satisfied

$$
\limsup _{t \rightarrow \infty} \frac{1}{t} \sum_{\tau=0}^{t-1} \mathbb{E}\left[P_{1}(\tau)\right] \leq \hat{P}_{1}
$$

and

$$
\lim \sup _{t \rightarrow \infty} \frac{1}{t} \sum_{\tau=0}^{t-1} \mathbb{E}\left[P_{2}(\tau)\right] \leq \hat{P}_{2}
$$

where $\mathbb{E}[\cdot]$ denotes expectation and $\hat{P}_{1}$ and $\hat{P}_{2}$ denote the average transmission power constraints for OW and RF subsystems respectively. In addition,

$$
\lim \sup _{t \rightarrow \infty} \frac{1}{t} \sum_{\tau=0}^{t-1} \mathbb{E}\left[f_{1}\left(P_{1}(\tau)\right)+f_{2}\left(P_{2}(\tau)\right)\right] \leq \hat{P},
$$

where $\hat{P}$ is the average electrical power consumed by hybrid transmitter, and $f_{1}(\cdot)$ and $f_{2}(\cdot)$ are non-decreasing functions with respect to the tranmission power levels, that determine the electrical power consumed by the OW and RF subsystems, respectively. In general, the structure of these functions depends on the technology employed by each transmission subsystem. A relevant measurement campaign, regarding the total power consumption of OW and RF transmission subsystems for specific transmission powers and technologies, has been performed in [5].

\section{Channel Model}

Due to the insertion of the CRC in every packet, the erasure channel model is used for describing both OW and RF channels. As erasure we define the event that the received power is below a pre-defined threshold that determines whether errors are detected [2]. Next, we describe the mode of operation of the OW and RF transmission subsystems and derive the erasure probabilities for each link.

1) Transmission through the OW subsystem: We consider an OW subsystem which employs intensity modulation at the transmitter and direct detection at the receiver. Assuming $P_{1}$ is the transmitted optical power level, the erasure probability of the OW link is given by

$$
\epsilon_{O W}\left(P_{1}\right)=\operatorname{Pr}\left\{h_{1} P_{1} \leq P_{\text {sens }}\right\}=\operatorname{Pr}\left\{h_{1} \leq \frac{P_{\text {sens }}}{P_{1}}\right\},
$$

where $h_{1}$ denotes the channel gain of the OW link, while $P_{\text {sens }}$ is the sensitivity of the photodetector which ensures that CRC is successfully decoded.

Due to atmospheric effects, the channel gain of the OW link can be modeled as

$$
h_{1}=\bar{h}_{1} \tilde{h}_{1},
$$

where $\bar{h}_{1}$ accounts for path loss due to weather effects and geometric spread loss, and $\tilde{h}_{1}$ represents irradiance fluctuations caused by atmospheric turbulence. The path loss coefficient can be calculated by combining the Beer Lambert's law [1] with the geometric loss formula [4, Eq. (9)], yielding

$$
\bar{h}_{1}=\left(\operatorname{erf}\left(\frac{\sqrt{\pi} D}{\sqrt{2} \theta_{T} L}\right)\right)^{2} \exp (-v L),
$$

where $2 D$ is the aperture diameter, $\theta_{T}$ is the optical beam's divergence angle (in $m \mathrm{rad}$ ), $L$ is the link distance (in $\mathrm{km}$ ), and $v$ is the weather dependent attenuation coefficient (in $1 / \mathrm{km}$ ). Under a wide range of atmospheric conditions, turbulence induced fading is statistically described by the well known Gamma-Gamma distribution, whose atmospheric parameters $\alpha$ and $\beta$ are calculated based on the link distance $L$, the diameter 
of the receiver aperture $2 D$, the weather dependent index of refraction $C_{n}^{2}$, and the wavelength of the optical carrier $\lambda_{1}$ [1].

Based on the above and using [8, Eq. (7)], the erasure probability of the OW link can be analytically evaluated by

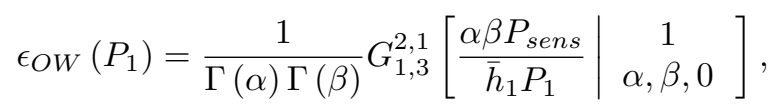

where $\Gamma(\cdot)$ is the Gamma function [9, Eq. 8.310)] and $G_{p, q}^{m, n}[\cdot]$ is the Meijer's $G$-function [9, Eq. (9.301)].

2) Transmission through the $R F$ subsystem: We will assume that the RF transmission subsystem uses a line-of-sight (LOS) link in the millimeter wave band. If $P_{2}$ is the transmitted RF power, the erasure probability of this subsystem is defined as

$$
\epsilon_{R F}\left(P_{2}\right)=\operatorname{Pr}\left\{\gamma_{R F} \leq \gamma_{t h}\right\},
$$

where $\gamma_{R F}$ is the instantaneous signal-to-noise ratio (SNR) at the RF receiver and $\gamma_{t h}$ is a threshold that ensures no errors are detected through the CRC. The instantaneous SNR of the $\mathrm{RF}$ link is defined as

$$
\gamma_{R F}=\frac{P_{2} g E_{s}}{\sigma_{2}^{2}} h_{2}^{2},
$$

where $h_{2}$ is the channel gain of the RF link, $g$ is the average path loss, $P_{2}$ is the transmitted RF power, $E_{s}$ is the average energy of the modulation alphabet, and finally $\sigma_{2}^{2}$ is the variance of the zero mean circularly symmetric complex Gaussian noise.

Assuming a carrier frequency of $60 \mathrm{GHz}$, the average path loss attenuation of the RF link is given by [10, Eq. (7)]

$$
g[d B]=G_{t}+G_{R}-20 \log _{10}\left(\frac{4 \pi L}{\lambda_{2}}\right)-\alpha_{o x y} L-\alpha_{\text {rain }} L,
$$

where $G_{t}$ and $G_{i}$ denote the transmit and receive antenna gains, respectively, $\lambda_{2}$ is the wavelength of the RF system, and $\alpha_{o x y}$ and $\alpha_{\text {rain }}$ are the attenuations caused by oxygen absorption and rain, respectively. Further, due to the LOS characteristic, the fading gain, $h_{2}$, is Ricean distributed [10], where the Ricean factor, $K$, depends on various link parameters, such as link distance and antenna height.

Based on the above and using [11, pp. 420], the erasure probability of the RF link can be analytically evaluated by

$$
\epsilon_{R F}\left(P_{2}\right)=1-Q_{1}\left(\sqrt{2 K}, \sqrt{\frac{2(1+K) \gamma_{t h}}{\frac{P_{2} g E_{s}}{\sigma_{2}^{2}}}}\right),
$$

where $Q_{1}(\cdot, \cdot)$ is the Marcum function [11, Eq. (4.34)].

\section{E. Available Controls and Queueing Dynamics}

Let $Q(t) \in\{1,2,3, \ldots\}$ represent the state of the queue of the hybrid transmitter at slot $t$. In each slot, the admission control decision determines $R(t)$, i.e., the number of new packets to admit into the queue, where

$$
0 \leq R(t) \leq A(t) .
$$

Further, in each slot, resource allocation decisions are made and $\left(P_{1}(t), P_{2}(t)\right)$ are determined from the sets $\mathcal{P}_{1}$ and $\mathcal{P}_{2}$, respectively. Based on these resource allocation decisions, the number of the packets that depart from the queue of the hybrid transmitter are determined. By removing a packet from the queue of the hybrid transmitter only when it is correctly received $^{1}$, the departures from the queue in each frame can be modeled as a random process $\mu\left(P_{1}(t), P_{2}(t)\right)$ which depends from the power levels $P_{1}(t)$ and $P_{2}(t)$ used for transmission by each subsystem. This process can be written as

$$
\mu\left(P_{1}(t), P_{2}(t)\right)=\mu_{1}\left(P_{1}(t)\right)+\mu_{2}\left(P_{2}(t)\right),
$$

where $\mu_{1}\left(P_{1}(t)\right)$ and $\mu_{2}\left(P_{2}(t)\right)$ are the stochastic processes that represent the numbers of packets transmitted from the OW and RF transmission subsystems respectively and are removed from the queue. These processes can be expressed as

$$
\mu_{1}\left(P_{1}(t)\right)=k \mathbb{1}_{1}\left(P_{1}(t)\right) \text { and } \mu_{2}\left(P_{2}(t)\right)=\mathbb{1}_{2}\left(P_{2}(t)\right),
$$

where $\mathbb{1}_{1}\left(P_{1}(t)\right)$ and $\mathbb{1}_{2}\left(P_{2}(t)\right)$ are indicator functions that represent the random success/failure transmission outcomes of each transmission subsystem, when the optical and RF transmission power levels are $P_{1}(t)$ and $P_{2}(t)$, respectively.

The probabilities of successful transmission for each subsystem, i.e., $\Phi_{1}\left(P_{1}(t)\right)=\mathbb{E}\left[\mathbb{1}_{1}\left(P_{1}(t)\right)\right]$ and $\Phi_{2}\left(P_{2}(t)\right)=$ $\mathbb{E}\left[\mathbb{1}_{2}\left(P_{2}(t)\right)\right]$, are assumed to be non-decreasing functions with respect to the transmission powers and are determined through the erasure probabilities of each link, i.e., $\Phi_{1}\left(P_{1}(t)\right)=1-\epsilon_{O W}\left(P_{1}(t)\right)$ and $\Phi_{2}\left(P_{2}(t)\right)=1-$ $\epsilon_{R F}\left(P_{2}(t)\right)$. Further, $k$ is a scaling parameter that accounts for the different transmission rates supported by each subsystem, i.e., $k=\frac{R_{O W}}{R_{R F}}$, where $R_{O W}$ and $R_{R F}$ are the transmission rates supported by the OW and RF subsystems, respectively. Since in most cases, OW systems can support higher data rates than their RF counterparts, we can assume that $k \geq 1$.

In view of the above, the queue evolves as [7]

$$
Q(t+1)=\left[Q(t)-\mu\left(P_{1}(t), P_{2}(t)\right)\right]^{+}+R(t),
$$

where $[x]^{+}=\max [x, 0]$.

\section{Control Objective And Transmission ALGORITHM}

The major design issue of the hybrid transmitter is to maximize its throughput by choosing the appropriate controls in each time-slot and ensuring that the average power constraints are satified. In what follows, we describe the stochastic optimization problem that arises for the system model under consideration and suggest a solution based on the well-known Lyapunov optimization framework [7].

\section{A. Control Objective}

Consider any control policy that makes admission control decisions $R(t)$ and power allocation $\left(P_{1}(t), P_{2}(t)\right)$. A control policy is called admissible if the queue of the hybrid transmitter is mean-rate stable, i.e., the output longterm rate of the queue should be equal with its input rate,

\footnotetext{
${ }^{1}$ Since most hybrid OW/RF links are bidirectional, acknowledgement for the correct reception at the transmitter can be obtained through piggybacking techniques.
} 
the constraint of the maximum number of admissible packets given by (13) is satisfied for every time-slot and the power constraints of (1)-(4) are satisfied. Under an admissible policy, the hybrid transmitter obtains a long-term average transmission throughput

$$
\bar{R}=\lim \inf _{t \rightarrow \infty} \frac{1}{t} \sum_{\tau=0}^{t-1} \mathbb{E}[R(\tau)] .
$$

Our goal is to select an admissible policy that maximizes the hybrid transmitter's throughput. Formally, this can be stated by the following stochastic optimization problem, named OPT1, as

$$
\begin{array}{cc}
\operatorname{max.} & \bar{R} \\
\text { s.t. } & \bar{R} \leq \bar{\mu} \\
& \text { Eqs. (1)-(4) } \\
& 0 \leq R(t) \leq A(t) \forall t \\
& P_{1}(t) \in \mathcal{P}_{1} \text { and } P_{2}(t) \in \mathcal{P}_{2} \forall t
\end{array}
$$

where

$$
\bar{\mu}=\lim \sup _{t \rightarrow \infty} \frac{1}{t} \sum_{\tau=0}^{t-1} \mathbb{E}\left[\mu\left(P_{1}(\tau), P_{2}(\tau)\right)\right] .
$$

We note that the conventional techniques to solve OPT1 are based on dynamic programming and require either extensive knowledge of the system dynamics or learning based approaches that suffer from large convergence times [12]. Therefore we apply the Lyapunov optimization framework [7] that is based only on the observations of the status of the queue in each time slot.

\section{B. Minimizing the "Drift Plus Penalty"}

In the Lyapunov optimization framework, a "Drift Plus Penalty" expression is derived that needs to be minimized [7]. For the optimization problem posed above, OPT1, this expression is derived in what follows.

In order to satisfy the long-term average power constraints of (2)-(4), three virtual queues are created, $H_{i}(t)$ with $i=$ $1,2,3$, which evolve as

$$
\begin{aligned}
& H_{1}(t+1)=\left[H_{1}(t)-\hat{P}_{1}\right]^{+}+P_{1}(t), \\
& H_{2}(t+1)=\left[H_{2}(t)-\hat{P}_{2}\right]^{+}+P_{2}(t)
\end{aligned}
$$

and

$$
H_{3}(t+1)=\left[H_{3}(t)-\hat{P}\right]^{+}+f_{1}\left(P_{1}(t)\right)+f_{2}\left(P_{2}(t)\right) .
$$

If the designed control policy makes both actual and virtual queues mean rate stable, all the constraints of OPT1 are satisfied.

Let $\Theta(t)=\left\{Q(t), H_{1}(t), H_{2}(t), H_{3}(t)\right\}$ represent the system queue backlog at slot $t$. As a measure of congestion in the system, we use the quadratic Lyapunov function, i.e.,

$$
L(\Theta(t))=\frac{1}{2} Q^{2}(t)+\frac{1}{2} \sum_{i=1}^{3} H_{i}^{2}(t) .
$$

Define the Lyapunov drift as the expected change in $L(\Theta(t))$ over the time-slot $t$ conditioned on $\Theta(t)$, i.e.,

$$
\Delta(\Theta(t))=\mathbb{E}[L(\Theta(t+1))-L(\Theta(t)) \mid \Theta(t)] .
$$

Due to (15) and (19)-(21), the Lyapunov drift can be upper bounded as

$$
\begin{aligned}
\Delta(\Theta(t)) & \leq B+\mathbb{E}\left[H_{1}(t)\left(P_{1}(t)-\hat{P}_{1}\right) \mid \Theta(t)\right] \\
& +\mathbb{E}\left[H_{2}(t)\left(P_{2}(t)-\hat{P}_{2}\right) \mid \Theta(t)\right] \\
& +\mathbb{E}\left[Q(t)\left(R(t)-\mu\left(P_{1}(t), P_{2}(t)\right)\right) \mid \Theta(t)\right] \\
& +\mathbb{E}\left[H_{3}(t)\left(f_{1}\left(P_{1}(t)\right)+f_{2}\left(P_{2}(t)\right)-\hat{P}\right) \mid \Theta(t)\right],
\end{aligned}
$$

where $B$ is a finite constant that satisfies for all $t$

$$
\begin{aligned}
B & \geq \frac{1}{2}\left(R^{2}(t)+\mu^{2}\left(P_{1}(t), P_{2}(t)\right)\right) \\
& +\frac{1}{2}\left(P_{1}^{2}(t)+\hat{P}_{1}^{2}\right)+\frac{1}{2}\left(P_{2}^{2}(t)+\hat{P}_{2}^{2}\right) \\
& +\frac{1}{2}\left(\hat{P}^{2}+\left(f_{1}\left(P_{1}(t)\right)+f_{2}\left(P_{2}(t)\right)\right)^{2}\right) .
\end{aligned}
$$

Taking into consideration (1), the fact that the functions $f_{1}(\cdot)$ and $f_{2}(\cdot)$ are non-decreasing, $R(t) \leq A_{\max }$ and $\mu\left(P_{1}(t), P_{2}(t)\right) \leq k+1$, it follows that the above equation can be satisfied by choosing

$$
\begin{aligned}
B=\frac{1}{2} & \left(A_{\text {max }}^{2}+(k+1)^{2}+P_{1, \text { max }}^{2}+P_{2, \text { max }}^{2}+\hat{P}_{1}^{2}\right. \\
& \left.+\hat{P}_{2}^{2}+\hat{P}^{2}+\left(f_{1}\left(P_{1, \max }\right)+f_{2}\left(P_{2, \max }\right)\right)^{2}\right) .
\end{aligned}
$$

By adding the penalty term $-V \mathbb{E}[R(t) \mid \Theta(t)]$ to both sides of (24), where $V$ is a utility-delay trade-off parameter, the "Drift Plus Penalty" expression of (27) is derived, which is given at the top of the next page.

Eq. (27) can be minimized by observing $\Theta(t)$ in every time slot and opportunistically minimizing expectation according to the Hybrid Transmitter Control (HTC) algorithm, described below.

\section{HTC Algorithm}

In each time-slot $t \in\{1,2,3, \ldots\}$, after observing $\Theta(t)$ do the following:

1) Admission Control: Choose the number of admitted packets according to

$$
R(t)= \begin{cases}A(t) & \text { if } Q(t) \leq V \\ 0 & \text { else }\end{cases}
$$

2) Resource Allocation: Choose the power allocation controls $\left(P_{1}(t), P_{2}(t)\right)$ that solve the following optimization problems:

$$
\begin{array}{cc}
\max . & k Q(t) \Phi_{1}\left(P_{1}(t)\right)-H_{1}(t) P_{1}(t) \\
& -H_{3}(t) f_{1}\left(P_{1}(t)\right) \\
\text { s.t. } & P_{1}(t) \in \mathcal{P}_{1}
\end{array}
$$




$$
\begin{aligned}
\Delta(\Theta(t))-V \mathbb{E}[R(t) \mid \Theta(t)] & \leq B+\mathbb{E}[(Q(t)-V) R(t) \mid \Theta(t)]-\mathbb{E}\left[Q(t) \mu\left(P_{1}(t), P_{2}(t)\right) \mid \Theta(t)\right] \\
& +\mathbb{E}\left[H_{1}(t)\left(P_{1}(t)-\hat{P}_{1}\right) \mid \Theta(t)\right]+\mathbb{E}\left[H_{2}(t)\left(P_{2}(t)-\hat{P}_{2}\right) \mid \Theta(t)\right] \\
& +\mathbb{E}\left[H_{3}(t)\left(f_{1}\left(P_{1}(t)\right)+f_{2}\left(P_{2}(t)\right)-\hat{P}\right) \mid \Theta(t)\right]
\end{aligned}
$$

and

$$
\begin{array}{cc}
\max & Q(t) \Phi_{2}\left(P_{2}(t)\right)-H_{2}(t) P_{2}(t) \\
& -H_{3}(t) f_{2}\left(P_{2}(t)\right) \\
\text { s.t. } & P_{2}(t) \in \mathcal{P}_{2}
\end{array}
$$

3) Queue Update: After choosing the appropriate controls, update queues according to (15), (19)-(21).

It should be noted that up to now we have assumed that both the channel statistics and the system parameters (such as link distance) remain constant over time slots. However, the proposed HTC algorithm can be applied in time-varying environments with minor changes; only by modifying the functions $\Phi\left(P_{1}(t)\right)$ and $\Phi\left(P_{2}(t)\right)$ in the per-slot decision rules of (29) and (30), accordingly.

\section{Performance Evaluation}

To analyze the performance of the HTC algorithm, we compare its Lyapunov drift with that of the optimal policy [7].

Theorem 1. Suppose the HTC algorithm is implemented over all time-slots $t \in\{1,2,3, \ldots\}$ with initial conditions $Q(0)=0$, $H_{1}(0)=H_{2}(0)=H_{3}(0)=0$ and with a control parameter $V>0$. Then:

1) The worst case queue backlog is upper bounded by a constant $Q_{\max }$ for all $t$ given by

$$
Q(t) \leq Q_{\max }=A_{\max }+V,
$$

2) The long-term time average throughput utility achieved by the HTC algorithm is within $\frac{B}{V}$ of the optimal value, i.e.,

$$
\bar{R} \geq v^{*}-\frac{B}{V},
$$

where $v^{*}$ denotes the optimal value of the objective in OPT1.

Proof: For the first part, suppose that $Q(t) \leq Q_{\max }$ at the frame $t$. We will show that the same holds at the frame $t+1$. If $Q(t) \leq Q_{\max }-A_{\max }$, then from (15) we have $Q(t+1) \leq$ $Q_{\max }$, since $R(t) \leq A(t) \leq A_{\max }$. Furthermore, if $Q(t) \geq$ $Q_{\max }-A_{\max }=V$, then the admission control part of the HTC algorithm chooses $R(t)=0$, resulting in $Q(t+1) \leq$ $Q(t) \leq Q_{\max }$. The second part can be directly derived by using the results of the Delayed Lyapunov Optimization with Rewards theorem presented in [13].

Theorem 1 shows that the long-term time-average throughput can be pushed to within $O\left(\frac{1}{V}\right)$ of the optimal value with a trade-off in the worst case queue backlog. By Little's Theorem, this leads to an $O\left(\frac{1}{V}, V\right)$ utility-delay tradeoff.

\begin{tabular}{|c|c|c|}
\hline \multicolumn{3}{|c|}{ OW sub-system } \\
\hline Parameter & Symbol & Value \\
\hline Data rate & $R_{O W}$ & $1 \mathrm{Gbps}$ \\
\hline Wavelength & $\lambda_{1}$ & $780 \mathrm{~nm}$ \\
\hline Max optical power & $P_{1, \max }$ & $40 \mathrm{~mW}$ \\
\hline Aperture diameter & $2 D$ & $10 \mathrm{~cm}$ \\
\hline Divergence angle & $\theta$ & $5 \mathrm{mrad}$ \\
\hline Receiver's Sensitivity & $P_{\text {sens }}$ & $1 \mu \mathrm{W}$ \\
\hline RF sub-system & Value \\
\hline Parameter & Symbol & $250 \mathrm{Mbps}$ \\
\hline Data rate & $R_{R F}$ & $60 \mathrm{GHz}$ \\
\hline Carrier frequency & $f_{c}$ & $10 \mathrm{~mW}$ \\
\hline Max transmit power & $P_{2, m a x}$ & $1 \mathrm{~J}$ \\
\hline Average symbol's energy & $E_{s}$ & $44 \mathrm{dBi}$ \\
\hline Transmit/Receive Antenna Gain & $G_{i}, G_{r}$ & $15.1 \mathrm{~dB} / \mathrm{km}$ \\
\hline Attenuation (oxygen) & $\alpha_{o x y}$ & $-85 \mathrm{dBm}$ \\
\hline Noise variance & $\sigma_{2}^{2}$ & $24 \mathrm{~dB}$ \\
\hline Threshold SNR & $\gamma_{t h}$ &
\end{tabular}

TABLE I

PARAMETERS OF THE HYBRID SYSTEM [2] AND [10].

\begin{tabular}{|c|c|c|c|}
\hline Weather conditions & $v(\mathrm{~dB} / \mathrm{km})$ & $\alpha_{\text {rain }}(\mathrm{dB} / \mathrm{km})$ & $C_{n}^{2}$ \\
\hline \hline Clear air & 0.43 & 0 & $5 \cdot 10^{-14}$ \\
\hline Haze & 4.2 & 0 & $1.7 \cdot 10^{-14}$ \\
\hline
\end{tabular}

TABLE II

WEATHER DEPENDENT PARAMETERS OF OW AND RF CHANNELS [10].

\section{Results \& Discussion}

In this section, we present numerical results that illustrate the performance of the presented algorithm under various weather conditions. For deriving these results, we assume that the link distance is $L=1500 \mathrm{~m}$, the sets of available transmission power levels are $\mathcal{P}_{1}=\left\{0, P_{1, \max }\right\}$ and $\mathcal{P}_{2}=\left\{0, P_{2, \max }\right\}, \hat{P}_{1}=P_{1, \max }$ and $\hat{P}_{2}=P_{2, \max }$, while the maximum average total power consumption at the transmitter is equal with $\hat{P}=90 \mathrm{~mW}$. Furthermore, the functions $f_{1}(\cdot)$ and $f_{2}(\cdot)$ are assumed to be given by

$$
f_{1}\left(P_{1}(t)\right)=\frac{P_{1}(\tau)}{\eta_{1}} \text { and } f_{2}\left(P_{2}(t)\right)=\frac{P_{2}(\tau)}{\eta_{2}}
$$

where $\eta_{1}$ and $\eta_{2}$ denote the wall-plug efficiency of the laser [14] and the power efficiency factor of the RF transmitter [15], respectively, and assumed to be equal with $\eta_{1}=40 \%$ and $\eta_{2}=50 \%$. The values for the remaining parameters of the respective OW and RF transmission subsystems and channel model are summarized in Tables I and II.

Fig. 2 illustrates the average throughput achieved by the HTC transmission algorithm under various weather conditions. Specifically, the average number of the packets admitted in the queue, $\bar{R}$, is plotted with respect to the mean rate of the 


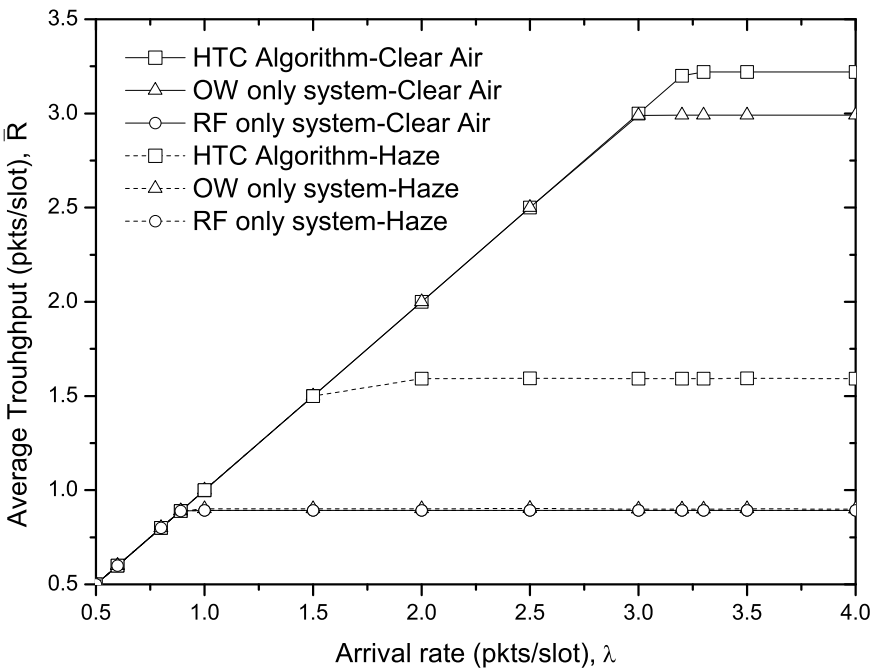

Fig. 2. Average throughput in clear air and haze weather conditions.

arrival process, assuming $V=100$ and clear air and haze weather conditions. As benchmarks, the throughputs achieved by the OW-only and RF-only systems, i.e., the OW and RF subsystems respectively that operate distinctly but have the same average transmission and total power constraints with the hybrid transmitter, are also plotted. It can be easily observed that the HTC algorithm is linear with respect to $\lambda$ until a threshold $\lambda^{*}$. This threshold was found to be within $\frac{B}{V}$ of the value achieved by the optimum policy. Furthermore, when compared with the throughputs achieved by the OW-only and RF-only systems, the HTC algorithm enhances the achievable throughput region of the hybrid transmitter without any additional power. This clearly demonstrates that the proposed transmission scheme efficiently exploits both $\mathrm{OW}$ and RF links.

The effects of $V$ parameter on the throughput achieved by the HTC algorithm are illustrated at Fig. 3, assuming clear air weather conditions. Although the increase of $V$ increases the queue backlog, it can be observed that it improves the performance of the proposed algorithm. Hence, with the appropriate selection for the parameter $V$, the performance of the HTC algorithm can be pushed arbitrary close to the optimal value.

\section{CONCLUSIONS}

We developed a transmission algorithm for hybrid OW/RF systems that maximizes their throughput subject to the transmitter's per-link and total power constraints. We used the Lyapunov Optimization framework to design an online flow control and resource allocation algorithm, which provides tight guarantees in terms of power consumption, while its performance can be pushed arbitrarily close to the optimal value with a trade-off in the average delay. In our ongoing research, we shall investigate reduced reverse feedback transmission algorithms for this power-constrained transmission scenario.

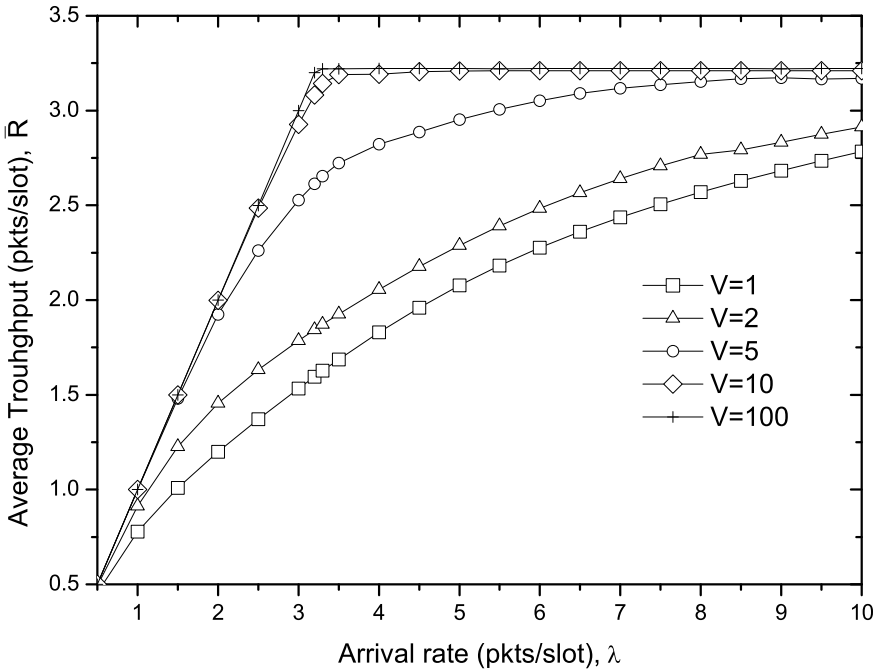

Fig. 3. The effects of the $V$ parameter on the achieved throughput assuming clear air weather conditions.

\section{REFERENCES}

[1] L. Andrews, R. L. Philips, and C. Y. Hopen, Laser Beam Scintillation with Applications. SPIE Press, 2001.

[2] S. Bloom and D. J. T. Heatley, "The last mile solution: Hybrid FSO radio," white paper, AirFiber Inc., 802-0008-000 M-A1, pp. 1-20, May 2002.

[3] D. K. Borah, A. C. Boukouvalas, C. C. Davis, and S. H. H. Yiannopoulos, "A review of communication-oriented optical wireless systems," EURASIP J. Wireless Commun. and Networking, vol. 91, 2012.

[4] W. Zhang, S. Hranilovic, and C. Shi, "Soft-switching hybrid FSO/RF links using short-length Raptor-codes: Design and implementation," IEEE J. Sel. Areas Commun., vol. 27, no. 9, pp. 1698-1708, 2009.

[5] S. Deng, J. Liao, Z. R. Huang, M. Hella, and K. Connor, "Wireless connections of sensor network using RF and free space optical links," in Proc. SPIE, vol. 6303, 2007.

[6] F. Nadeem, S. Chessa, E. Leitgeb, and S. Zaman, "The effects of weather on the life time of wireless sensor networks using FSO/RF communication," Radioengineering, vol. 19, no. 2, pp. 262-270, 2010.

[7] M. J. Neely, Stochastic Network Optimization with Application to Communication \& Queueing Systems. Morgan \& Claypool, Aug. 2010.

[8] T. A. Tsiftsis, "Performance of heterodyne wireless optical communication systems over gamma-gamma atmospheric turbulence channels," Electronics Letters, vol. 44, no. 5, 2008.

[9] I. S. Gradshteyn and I. M. Ryzhik, Table of Integrals, Series, and Products, 6th ed. New York: Academic, 2000.

[10] B. He and R. Schober, "Bit-interleaved coded modulation for hybrid RF/FSO systems," IEEE Trans. Commun., vol. 57, no. 12, pp. 3753$3763,2009$.

[11] M. K. Simon and M.-S. Alouini, Digital communications over Fading Channels. Wiley Interscience, 2005.

[12] D. P. Bertsekas, Dynamic Programming and Optimal Control. MA: Athena Scientific, 2007, vol. vols. 1 \& 2 .

[13] R. Urgaonkar and M. J. Neely, "Opportunistic scheduling with reliability guarantees in cognitive radio networks," IEEE Trans. Mobile Comput., vol. 8, pp. 766-777, Jan 2009.

[14] G. P. Agrawal, Fiber-Optic Communication Systems. John Wiley \& Sons, Inc., 2002.

[15] J. N. Murdock and T. S. Rappaport, "Consumption factor and powerefficiency factor: A theory for evaluating the energy efficiency of cascaded communication systems," IEEE J. Sel. Areas Commun., vol. 32 no. 12, pp. 1-16, Dec. 2014. 